\title{
Telomere length and physical performance among older people pÿ the Helsinki Birth Cohort Study
}

\section{Åström, Max J.}

2019-10

Åström , M J , von Bonsdorff , M B , Perälä , M-M , Salonen , M K , Rantanen , T , Kajantie , E , Simonen , M , Pohjolainen, P , Haapanen , M J , Guzzardi , M A , lozzo , P , Kautiainen , pÿH \& Eriksson , J G 2019 , ' Telomere length and physical performance among older people the Helsinki Birth Cohort Study ', Mechanisms of Ageing and Development, vol. 183 , 111145 . https://doi.org/10.1016/j.mad.2019.111145

http://hdl.handle.net/10138/319020

https://doi.org/10.1016/j.mad.2019.111145

cc_by_nc_nd

acceptedVersion

Downloaded from Helda, University of Helsinki institutional repository.

This is an electronic reprint of the original article.

This reprint may differ from the original in pagination and typographic detail.

Please cite the original version. 


\section{Journal Pre-proof}

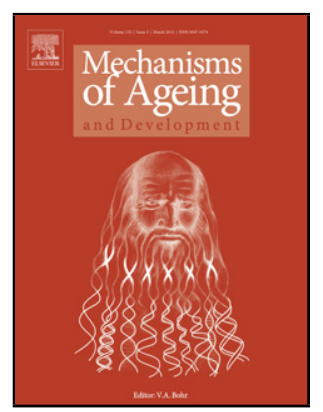

Telomere length and physical performance among older people - the Helsinki Birth Cohort Study

Max J. Åström, Mikaela B. von Bonsdorff, Mia-Maria Perälä, Minna K. Salonen, Taina Rantanen, Eero Kajantie, Mika Simonen, Pertti Pohjolainen, Markus J. Haapanen, Maria A. Guzzardi, Patricia Iozzo, Hannu Kautiainen, Johan G. Eriksson

PII: S0047-6374(19)30150-2

DOI: https://doi.org/10.1016/j.mad.2019.111145

Reference: MAD 111145

To appear in: Mechanisms of Ageing and Development

Received Date: 3 May 2019

Revised Date: 26 August 2019

Accepted Date: 29 August 2019

Please cite this article as: Åström MJ, von Bonsdorff MB, Perälä M-Maria, Salonen MK, Rantanen T, Kajantie E, Simonen M, Pohjolainen P, Haapanen MJ, Guzzardi MA, lozzo P, Kautiainen $\mathrm{H}$, Eriksson JG, Telomere length and physical performance among older people the Helsinki Birth Cohort Study, Mechanisms of Ageing and Development (2019), doi: https://doi.org/10.1016/j.mad.2019.111145 
This is a PDF file of an article that has undergone enhancements after acceptance, such as the addition of a cover page and metadata, and formatting for readability, but it is not yet the definitive version of record. This version will undergo additional copyediting, typesetting and review before it is published in its final form, but we are providing this version to give early visibility of the article. Please note that, during the production process, errors may be discovered which could affect the content, and all legal disclaimers that apply to the journal pertain.

(C) 2019 Published by Elsevier. 


\section{Telomere length and physical performance among older people - the Helsinki Birth Cohort Study}

Max J. Åströmª, , Mikaela B. von Bonsdorff ${ }^{\mathrm{b}, \mathrm{c}}$, Mia-Maria Perälä ${ }^{\mathrm{b}, \mathrm{d}}$, Minna K. Salonen ${ }^{\mathrm{b}, \mathrm{d}}$, Taina Rantanen $^{\mathrm{c}}$, Eero Kajantie ${ }^{\mathrm{d}-\mathrm{g}}$, Mika Simonen ${ }^{\mathrm{h}}$, Pertti Pohjolainen ${ }^{\mathrm{i}}$, Markus J. Haapanen ${ }^{\mathrm{a}, \mathrm{b}}$, Maria A. Guzzardi $^{\mathrm{j}}$, Patricia Iozzo ${ }^{\mathrm{j}}$, Hannu Kautiainen ${ }^{\mathrm{a}, \mathrm{b}}$, Johan G. Eriksson ${ }^{\mathrm{a}, \mathrm{b}, \mathrm{d}, \mathrm{k}, \mathrm{l}}$

${ }^{a}$ Department of General Practice and Primary Health Care, University of Helsinki and Helsinki University Hospital, Helsinki, Finland

${ }^{\mathrm{b}}$ Folkhälsan Research Center, Helsinki, Finland

${ }^{\mathrm{c}}$ Faculty of Sport and Health Sciences and Gerontology Research Center, University of Jyväskylä, Jyväskylä, Finland

${ }^{d}$ Department of Public Health Solutions, Public Health Promotion Unit, National Institute for Health and Welfare, Helsinki, Finland

${ }^{\text {e }}$ PEDEGO Research Unit, MRC Oulu, Oulu University Hospital and University of Oulu, Oulu, Finland

${ }_{\mathrm{f}}^{\mathrm{f}}$ Department of Clinical and Molecular Medicine, Norwegian University of Science and Technology, Trondheim, Norway

${ }^{\mathrm{g}}$ Hospital for Children and Adolescents, Helsinki University Central Hospital and University of Helsinki, Helsinki, Finland

${ }^{\text {h }}$ Faculty of Arts, University of Helsinki, Helsinki, Finland

${ }^{\mathrm{i}}$ Age Institute, Helsinki, Finland

j Institute of Clinical Physiology, National Research Council (CNR), Pisa, Italy

${ }^{k}$ Singapore Institute for Clinical Sciences (SICS), Agency for Science, Technology and Research (A*STAR), Singapore.

${ }^{1}$ Obstetrics \& Gynecology, Yong Loo Lin School of Medicine, National University of Singapore and National University Health System, Singapore.

Corresponding Author:

Max Åström, MD

Department of General Practice and Primary Health Care

University of Helsinki

PO Box 20

FI-00014 University of Helsinki

max.astrom@helsinki.fi

\section{Highlights}

- There was an association between telomere length and physical performance

- Greater telomere attrition was associated with poorer physical performance

- Results were significant only for women

- Telomeres could potentially be used as a biomarker of physical performance 


\begin{abstract}
Telomere length has been suggested a biomarker of aging and is associated with several chronic diseases. However, the association between telomere length and physical performance is not well known. Using both cross-sectional and longitudinal data, we studied 582 women and 453 men from the Helsinki Birth Cohort Study at two time-points; a baseline examination in 2001-2004 at a mean age of 61 years and a follow-up examination approximately 10 years later in 2011-2013. Telomere length was measured both at baseline and at follow-up using real-time quantitative polymerase chain reaction. Physical performance was evaluated only at follow-up using the Senior Fitness Test (SFT), which assesses strength, flexibility and endurance. In women, shorter telomere length at follow-up $(p=0.044)$ and greater telomere attrition during follow-up time $(p=0.022)$ were associated with poorer physical performance after adjusting for covariates (age at baseline, smoking status, body mass index at baseline, follow-up time and educational attainment). No similar associations were found for men. This indicates that, at least in women, telomere length could potentially be used as a biomarker for physical performance, however, more longitudinal studies are needed to confirm this association.
\end{abstract}

Keywords: Biomarkers, Physical function, Aging, Epidemiology

\author{
Abbreviations \\ HBCS, Helsinki Birth Cohort Study; LTPA, leisure-time physical activity; MET, metabolic \\ equivalent of task; SFT, Senior Fitness Test
}

\title{
1. Introduction
}

Aging can be defined as a gradual event causing decline in biological functions and is a major risk factor for both morbidity and mortality (Simm et al., 2008). However, due to the heterogeneity of the aging process, chronological age is a poor indicator of health and function (Mitnitski et al., 2002). Therefore, studies from the past decade have used other methods for assessing the biological age of an individual (Martin-Ruiz et al., 2011). Telomere length has been proposed as one potential 
biomarker of aging (von Zglinicki and Martin-Ruiz, 2005). Telomeres are repetitive nucleoprotein complexes at the ends of eukaryotic chromosomes that protect functional DNA (Blackburn, 2001). Telomeres shorten at each cell division due to incomplete DNA replication at chromosome ends, however, the rate of telomere attrition is further induced by inflammation and oxidative stress as well as e.g. smoking and obesity (Lorenzi et al., 2018). Telomere length has been shown to correlate inversely with age and several age-related diseases including type 2 diabetes, cardiovascular disease, renal failure and Alzheimer's disease (Bernadotte et al., 2016). The association between telomere length and mortality has been inconsistent in previous studies (Cawthon et al., 2003; Martin-Ruiz et al., 2005).

Among older people, physical performance is an important indicator of both current and future health status. With decreased physical function, the risk of morbidity such as cardiovascular disease and cognitive decline increases, and poor physical performance also predicts future hospitalization and institutionalization (Cooper et al., 2010a). In community-dwelling populations, objectively measured poor physical performance has been shown to increase the risk of all-cause mortality (Cooper et al., 2010b). Aging is associated with a loss of muscle mass, known as sarcopenia, which is an important reason for decreased physical function among older people (Legrand et al., 2014). To avoid rises in health care costs (Fried et al., 2001) there is an inevitable need for finding ways to predict and prevent decline in physical performance at an early stage among older people.

The association between telomere length and physical performance is not well known. Only little evidence is available and the results have been inconsistent, most having shown no association between the two variables (Mather et al., 2010; Maynard et al., 2015). Cross-sectional studies have assessed telomere length at only one time-point thereby unable to report any change in telomere length over time. As inflammation and oxidative stress are associated with both decreased physical performance and increased rate of telomere attrition, an attractive hypothesis is that changes in 
telomere length better predicts subsequent physical decline compared to using a single measure of telomere length. Previous longitudinal studies have assessed physical performance using simple test batteries only assessing limited aspects of physical performance, such as grip strength or standing balance (Gardner et al., 2013). These tests may thereby not give an accurate representation of the overall physical performance of the individuals.

The aim of this study was to assess if telomere length was related to objectively measured physical performance 10 years later. We also assessed if telomere attrition over a 10-year period or telomere length in cross-sectional analysis was associated with physical performance. We studied individuals from the Helsinki Birth Cohort Study at an average age of 61 years and assessed physical performance with the validated Senior Fitness Test (SFT).

\section{Material and methods}

\subsection{Study population}

This study is part of the Helsinki Birth Cohort Study (HBCS) and uses data from a subpopulation of the HBCS consisting of 8760 individuals born between 1934 and 1944 at the Helsinki University Central Hospital. All subjects visited child welfare clinics in Helsinki and were residents of Finland in 1971, when they received a unique personal identification number (Åström et al., 2018). In the year 2000, random-number tables were used to invite 2902 individuals to a baseline clinical examination, of which 2003 cohort members participated in an examination between 2001 and 2004 . From this clinical cohort, those still alive and living within a $100-\mathrm{km}$ radius of Helsinki were invited to a follow-up examination in 2011. A total of 1097 of the invited 1404 cohort members participated in this follow-up examination between 2011 and 2013. The present study sample consists of 1035 cohort members with adequate data on both telomere length and physical performance. The clinical 
study protocol was approved by the Coordinating Ethics Committee of the Hospital District of Helsinki and Uusimaa and follows the guidelines of the Declaration of Helsinki. A written, informed consent was obtained from all participants.

\subsection{DNA extraction and telomere length}

DNA was extracted from EDTA-anti-coagulated peripheral whole blood both at the baseline clinical examination in 2001-2004 and at the clinical follow-up examination in 2011-2013 using a commercially available kit, QIAamp Blood Maxi Kit and DNeasy Blood \& Tissue Kit (Qiagen, The Netherlands), respectively. The samples were stored at $-20^{\circ} \mathrm{C}$ before and after DNA extraction. DNA concentration and purity were assessed by measuring ultraviolet absorbance at $260 \mathrm{~nm}$ for salts and $280 \mathrm{~nm}$ for other contaminants. DNA integrity was tested by electrophoresis in $0.8 \%$ agarose gel. There was no difference in DNA integrity and quality between the two kits.

Telomere length was assessed in leukocytes and measured using real-time quantitative polymerase chain reaction (PCR) as previously described (Guzzardi et al., 2015). At the baseline examination, telomere length was determined as the ratio of telomere DNA intensity to hemoglobin beta singlecopy gene signal intensity (Cawthon, 2002; Kajantie et al., 2012; O'Callaghan et al., 2008). A multiplex real-time quantitative PCR using a 384-well plate (CFX384 Touch Real-Time PCR Detection System, Bio-Rad Laboratories, USA) was used to measure telomere length at the followup examination (Cawthon, 2009; Guzzardi et al., 2015). All plates included 3 or 4 genomic DNA control samples for calibrating the plate effect and for monitoring the inter-assay coefficient of variation, which was $21.0 \%$ at baseline and $6.2 \%$ at the follow-up examination. Telomere length at both time points correlated with each other $(r=0.255, \mathrm{p}<0.001)$.

Telomere length is expressed as a T/S ratio, calculated as the ratio of telomere repeat copy number (T) to single gene copy number $(\mathrm{S})$ for each sample compared to a reference sample. Absolute change 
in telomere length during follow-up was calculated by subtracting baseline telomere length from follow-up telomere length. Relative telomere length after the follow-up period was calculated as follow-up telomere length divided by telomere length at baseline.

\subsection{Physical performance}

Physical performance was assessed at the follow-up examination in 2011-2013 using the validated SFT (Rikli and Jones, 1999). A modified test battery consisting of 5 tests was used to objectively evaluate the level of physical fitness: 1) Arm curl: holding hand weight (2kg for women and $3 \mathrm{~kg}$ for men), number of full-range biceps curls during 30-s to assess upper-body strength; 2) Chair stand: number of full chair stands during 30-s with arms folded across chest to evaluate lower-body strength; 3) Chair sit-and-reach: sitting at the front of the chair with legs fully extended, distance (in centimeters) between extended fingers and tip of toes to assess lower-body flexibility; 4) Back scratch: number of centimeters between tip of extended fingers, with one hand reaching over shoulder and the other hand up middle of back to evaluate upper-body flexibility; 5) 6-min walk: number of meters walked in 6 minutes to assess aerobic endurance. All tests were performed at a research facility and measurements were done by trained research assistants.

For each test, sex-standardized percentile tables of normative data for 5-year age groups were used to rate the score of the participants (Rikli and Jones, 2013). Based on 5-percentile ranges, the rating for each test varied between 1 and 20, those with a score in the lowest 5-percentiles receiving a rating of 1 and those with a score in the highest 5-percentiles receiving a rating of 20. The overall SFT score was then calculated by summing the normalized ratings of the SFT test components and varied between 5 and 100 .

\subsection{Study variables and covariates}


At the baseline examination, height and weight were measured and body mass index (BMI) was calculated as $\mathrm{kg} / \mathrm{m}^{2}$. Information on current health status, disease history, smoking status, level of education and lifestyle characteristics were acquired using questionnaires. Blood was drawn after fasting in order to measure glucose and lipids. The Kuopio Ischaemic Heart Disease Risk Factor Study (KIHD) 12-month leisure-time physical activity (LTPA) history questionnaire was used to assess physical activity (Lakka and Salonen, 1992). This validated exercise questionnaire assigns a metabolic equivalent of task (MET) value based on intensity grade for the most common LTPA and assesses physical activity during the last 12 months. The results are presented as MET-hours per year.

\subsection{Statistical analysis}

Data are presented as means with standard deviations (SD) or as counts with percentages. The study population was divided into tertiles based on the overall SFT score. In univariate analysis, linearity across SFT tertiles for baseline variables and telomere measurements were evaluated using the Cochran-Armitage test for trend and analysis of variance (ANOVA) with an appropriate contrast. In the adjusted model, linearity (orthogonal polynomial) of telomere measurements across SFT tertiles was evaluated using analysis of covariance (ANCOVA) and regression analysis. In the case of violation of the assumptions (e.g. non-normality), a bootstrap-type test was used. In the adjusted model, we controlled for age at baseline, smoking status, BMI at baseline, follow-up time and years of education. A possible nonlinear relationship between SFT and relative telomere length at followup was assessed using 3-knot-restricted cubic spline regression. The length of the distribution of knots were located at the 10th, 50th, and 90th percentiles; knot locations were based on Harrell's recommended percentiles or user-specified points (Harrell Jr., 2001). Correlation coefficients with 95\% CI were calculated using the Spearman method. The normality of the variables was tested using the Shapiro-Wilk W test. Stata 15.1 (StataCorp LP; College Station, Texas, USA) statistical package was used for the analysis. 


\section{Results}

Characteristics for the 582 women and 453 men included in the analysis are shown in Table 1. Baseline measurements were assessed at a mean age of 61 (SD 3) years. Physical performance was measured 9.7 years later (range 7.7 to 11.5 years). For both men and women, those with the highest BMI, highest glucose and triglyceride levels as well as the lowest HDL cholesterol fell into the lowest tertile of the SFT. The prevalence of cardiovascular disease and diabetes were highest in the lowest SFT tertile, whereas individuals with the highest education had the best physical performance, for both men and women. In addition, smoking status showed a negative association and LTPA a positive association across SFT tertiles for women but not men.

Mean telomere length T/S ratio at baseline was 1.42 (SD 0.29) for women and 1.38 (SD 0.29) for men, whereas the corresponding values at follow-up were 0.89 (SD 0.32) and 0.81 (SD 0.27) for women and men, respectively. Table 2 presents the unadjusted associations between telomere length measurements and SFT score tertiles. Telomere length at baseline was not associated with the SFT score 10 years later for neither women $(p=0.87)$ nor men $(p=0.45)$. At follow-up, however, longer telomere length was associated with a higher overall SFT score for women $(p=0.021)$ but not for men $(\mathrm{p}=0.56)$. Adjustment for age at baseline, smoking status, BMI at baseline, follow-up time and years of education attenuated the association but it remained statistically significant for women $(\mathrm{p}=0.044)$ and non-significant for men $(\mathrm{p}=0.13)$. Greater absolute telomere change between baseline and followup examinations was also associated with a lower overall SFT score, but only for women $(\mathrm{p}=0.039)$. Adjusting for age at baseline, smoking status, BMI at baseline, follow-up time and years of education did not attenuate this association $(\mathrm{p}=0.022)$. The corresponding $\mathrm{p}$-values for men were $\mathrm{p}=0.86$ (crude) and $p=0.65$ (adjusted). Similarly, there was a linear association between relative telomere length at 
follow-up and the SFT score for women but not for men. No correlation was found between telomere measurements and individual SFT components in neither women nor men (Table 3).

Figure 1 shows a scatter-plot on the association between baseline telomere length and SFT at followup. Baseline telomere length was not related to physical performance at follow-up in women ( $\beta=-$ $0.02,95 \% \mathrm{CI}-0.10$ to 0.05$)$ or in men $(\beta=0.00,95 \% \mathrm{CI}-0.09$ to 0.09$)$. The corresponding coefficients for the association between follow-up telomere length and physical performance for women $(\beta=-0.09$, $95 \% \mathrm{CI} 0.00$ to 0.17$)$ and men $(\beta=0.04,95 \% \mathrm{CI}-0.05$ to 0.13$)$ are shown in figure 2 . The association between SFT score and relative telomere length after follow-up is illustrated in Figure 3. Greater relative telomere length was associated with better physical performance, but only in women.

\section{Discussion}

In this cohort study of older people, we found a linear association between telomere length and physical performance among women in cross-sectional analysis. No similar association was observed for men. In addition, greater change in telomere length during the 10-year follow-up correlated with poorer physical performance among women but not men. Controlling for BMI, smoking and years of education did not attenuate the associations.

Ideally, telomere length could be used as a marker to assess and predict multicomplex age-related conditions, including decline in physical performance. However, findings in previous studies on the association between telomere length and physical performance have been inconsistent. In a metaanalysis by Gardner and colleagues (Gardner et al., 2013) comprising 4 studies, only chair rise speed and grip strength showed weak associations with telomere measurements. However, telomere length did not correlate with the other tests of physical performance, including walking speed and balance. Similarly, the Cardiovascular Health Study found a positive association between telomere length and 
chair stand result (Soares-Miranda et al., 2015). Some studies have also assessed the association between telomere length and frailty. Our earlier findings from the Helsinki Birth Cohort Study showed an inverse association between telomere length and risk of frailty (Haapanen et al., 2018). In contrast, a meta-analysis consisting of 3268 participants found no association between the two variables (Zhou et al., 2018).

Telomere length can be linked to physical performance by both direct and indirect pathways. When telomeres reach a critical point of shortening (less than 200-300 base pairs), cell cycle arrest, cellular senescence and apoptosis are triggered, which may cause loss of functional tissue (Aubert and Lansdorp, 2008). Thus, loss of muscle mass and muscle function due to telomere attrition could be a cause of poor physical performance (Zaslavsky et al., 2013). Another possible mechanism is oxidative stress and chronic inflammation, which has been shown to correlate with both telomere attrition (Lorenzi et al., 2018) and poor physical performance among older people (Cesari et al., 2004). This explanation is further supported by previous findings showing that senescent cells also secrete inflammatory mediators, such as interleukin-6 (Rodier et al., 2009).

Several reasons may account for the inconsistent findings in previous studies on the association between telomere length and physical performance. An obvious reason is differences in sample size, as smaller studies may not have enough statistical power to show an association. Another reason for discrepancy between results may be the technique used for measuring telomere length. The interindividual variation of telomere length is large and quantitative PCR has been shown to have a high coefficient of variation (Martin-Ruiz et al., 2015). If follow-up time in longitudinal studies is inadequate, this may lead to undetectable changes in telomere length and thereby non-significant results. Finally, the age-range of the study population could affect the results, as telomere length may 
be a stronger predictor of health outcomes for younger adults compared to older people (Brown et al., 2018).

We found that telomere attrition and telomere length associated with physical performance only among women, and this may be due to several factors. There were greater differences in lifestyle characteristics among women with better physical performance compared to men, regarding e.g. smoking and prevalence of cardiovascular disease, which could explain the difference observed in the association between the sexes. Besides evaluating muscle strength, the SFT used in our study also tests for flexibility, which may be another reason we found a relationship between the overall SFT score and physical performance only in women. Previous studies have generally reported longer telomere length in women (Woo et al., 2008; Yu et al., 2015), which may be due to hormonal differences between the sexes. Estrogen has been shown to upregulate telomerase activity, which increases the regeneration potential of telomeres (Kyo et al., 1999). In addition, estrogen reduces the burden of oxidative stress, thereby decreasing the rate of telomere attrition (Aviv, 2002). On the other hand, longer telomeres at baseline have been shown to correlate with a higher telomere attrition rate (Nordfjäll et al., 2009). Thus, although women have slower telomere attrition at younger age, the longer telomere length at older age may cause greater relative change in telomere length compared to men, when studying older people.

The strengths of this study are numerous. In this longitudinal study we included over 1000 wellphenotyped men and women who were followed-up for almost 10 years. Physical performance was assessed objectively using the validated SFT, which can be considered an appropriate method for measuring physical performance. SFT evaluates strength, flexibility and endurance, all important components of physical function. Furthermore, we measured telomere length at two time points, 
which allowed us to investigate how telomere attrition over time correlates with physical performance.

A weakness of this study is that we did not measure physical performance at baseline. We were therefore not able to evaluate a potential change in physical performance during follow-up and investigate if this correlates with either telomere length or telomere attrition. We found no association between baseline telomere length and physical performance 10 years later, thus, the follow-up time needed for predictive results remains unclear. Although the SFT measures overall physical performance, it should be noted that $40 \%$ of the score consists of measures on flexibility but no exhaustive test on endurance is included. This warrants further studies on the topic as different performance test batteries may reveal other kind of associations. We were able to account for several covariates in the analyses including BMI and smoking but acknowledge the possibility of other unmeasured variables affecting our results. We assessed telomere length from peripheral blood leukocytes, however, we cannot exclude that telomere length measured from e.g. muscle cells would better correlate with physical performance. Telomere length has been shown to have high heritability (Broer et al., 2013) and our study consisted of a homogenous group of participants living in a restricted area of Finland. This should be considered before generalizing our results.

In conclusion, we found evidence suggesting that shorter telomere length in cross-sectional analysis and greater telomere attrition over a 10 -year period were associated with poorer physical performance among older women. However, further longitudinal studies with sufficient follow-up times and larger study populations assessing physical performance at all time points are needed to confirm these findings in order to evaluate the usability of telomere length as a biomarker for physical performance.

\section{Acknowledgements}


Declarations of interest: none.

Author contributions: MA, MBvB, HK and JGE conceived and designed the study. JGE, EK, MKS, MBvB, PP, MS, TR, MH, MG, PI and MP planned and participated in data collection. HK carried out the statistical analysis. MA, MBvB, HK and JGE interpreted the data. MÅ wrote the first draft of the manuscript. All authors contributed to the critical review of the manuscript and have approved the final manuscript.

Funding: This work was supported by Emil Aaltonen Foundation; Finnish Foundation for Cardiovascular Research; Finnish Foundation for Diabetes Research; Finnish Foundation for Pediatric Research; Juho Vainio Foundation; Novo Nordisk Foundation; Signe and Ane Gyllenberg Foundation; Samfundet Folkhälsan; Finska Läkaresällskapet; Liv och Hälsa; European Commission FP7 DORIAN [grant number 278603]; EU H2020-PHC-2014-DynaHealth [grant number 633595]; and Academy of Finland [grant numbers 257239 to MBvB, 127437 to EK, 129306 to EK, 130326 to EK, 134791 to EK, 263924 to EK, 274794 to EK, 129369 to JGE, 129907 to JGE, 135072 to JGE, 129255 to JGE, 126775 to JGE].

Sponsor's role: Sponsors had no role in the design, methods, subject recruitment, data collections, analysis or preparation of this paper. 


\section{References}

Åström, M.J., von Bonsdorff, M.B., Perälä, M.M., Salonen, M.K., Rantanen, T., Kajantie, E., Simonen, M., Pohjolainen, P., Osmond, C., Eriksson, J.G., 2018. Glucose regulation and physical performance among older people: the Helsinki Birth Cohort Study. Acta Diabetol. 55, 1051-1058.

Aubert, G., Lansdorp, P.M., 2008. Telomeres and aging. Physiol Rev. 88, 557-579.

Aviv, A., 2002. Telomeres, sex, reactive oxygen species, and human cardiovascular aging. J Mol Med (Berl). 80, 689-695.

Bernadotte, A., Mikhelson, V.M., Spivak, I.M., 2016. Markers of cellular senescence. Telomere shortening as a marker of cellular senescence. Aging (Albany NY). 8, 3-11.

Blackburn, E.H., 2001. Switching and signaling at the telomere. Cell. 106, 661-673.

Broer, L., Codd, V., Nyholt, D.R., Deelen, J., Mangino, M., Willemsen, G., Albrecht, E., Amin, N., Beekman, M., De Geus, E.J., 2013. Meta-analysis of telomere length in 19713 subjects reveals high heritability, stronger maternal inheritance and a paternal age effect. Eur J Hum Genet. 21, 1163-1168.

Brown, L.L., Zhang, Y.S., Mitchell, C., Ailshire, J., 2018. Does Telomere Length Indicate Biological, Physical, and Cognitive Health Among Older Adults? Evidence from the Health and Retirement Study. J Gerontol A Biol Sci Med Sci. 73, 1626-1632.

Cawthon, R.M., 2002. Telomere measurement by quantitative PCR. Nucleic Acids Res. 30, e47.

Cawthon, R.M., 2009. Telomere length measurement by a novel monochrome multiplex quantitative PCR method. Nucleic Acids Res. 37, e21.

Cawthon, R.M., Smith, K.R., O'Brien, E., Sivatchenko, A., Kerber, R.A., 2003. Association between telomere length in blood and mortality in people aged 60 years or older. Lancet. 361, 393-395. 
Cesari, M., Penninx, B.W., Pahor, M., Lauretani, F., Corsi, A.M., Williams, G.R., Guralnik, J.M., Ferrucci, L., 2004. Inflammatory markers and physical performance in older persons: the InCHIANTI study. J Gerontol A Biol Sci Med Sci. 59, 242-248.

Cooper, R., Kuh, D., Cooper, C., Gale, C.R., Lawlor, D.A., Matthews, F., Hardy, R., FALCon and HALCyon Study Teams, 2010a. Objective measures of physical capability and subsequent health: a systematic review. Age Ageing. 40, 14-23.

Cooper, R., Kuh, D., Hardy, R., Mortality Review Group, FALCon and HALCyon Study Teams, 2010b. Objectively measured physical capability levels and mortality: systematic review and metaanalysis. BMJ. 341, c4467.

Fried, T.R., Bradley, E.H., Williams, C.S., Tinetti, M.E., 2001. Functional disability and health care expenditures for older persons. Arch Intern Med. 161, 2602-2607.

Gardner, M.P., Martin-Ruiz, C., Cooper, R., Hardy, R., Sayer, A.A., Cooper, C., Deary, I.J., Gallacher, J., Harris, S.E., Shiels, P.G., 2013. Telomere length and physical performance at older ages: an individual participant meta-analysis. PLoS One. 8, e69526.

Guzzardi, M.A., Iozzo, P., Salonen, M., Kajantie, E., Eriksson, J.G., 2015. Rate of telomere shortening and metabolic and cardiovascular risk factors: A longitudinal study in the 1934-44 Helsinki Birth Cohort Study. Ann Med. 47, 499-505.

Haapanen, M.J., Perälä, M.-M., Salonen, M.K., Guzzardi, M.A., Iozzo, P., Kajantie, E., Rantanen, T., Simonen, M., Pohjolainen, P., Eriksson, J.G., von Bonsdorff, M.B., 2018. Telomere Length and Frailty: The Helsinki Birth Cohort Study. J Am Med Dir Assoc. 19, 658-662.

Harrell Jr., F.E., 2001. Regression Modeling Strategies: With Applications to Linear Models, Logistic Regression, and Survival Analysis. Springer, New York. 
Kajantie, E., Pietiläinen, K.H., Wehkalampi, K., Kananen, L., Räikkönen, K., Rissanen, A., Hovi, P., Kaprio, J., Andersson, S., Eriksson, J.G., 2012. No association between body size at birth and leucocyte telomere length in adult life-evidence from three cohort studies. Int J Epidemiol. 41, 1400-1408.

Kyo, S., Takakura, M., Kanaya, T., Zhuo, W., Fujimoto, K., Nishio, Y., Orimo, A., Inoue, M., 1999. Estrogen activates telomerase. Cancer Res. 59, 5917-5921.

Lakka, T.A., Salonen, J.T., 1992. Intra-person variability of various physical activity assessments in the Kuopio Ischaemic Heart Disease Risk Factor Study. Int J Epidemiol. 21, 467-472.

Legrand, D., Vaes, B., Mathei, C., Adriaensen, W., Van Pottelbergh, G., Degryse, J.M., 2014. Muscle strength and physical performance as predictors of mortality, hospitalization, and disability in the oldest old. J Am Geriatr Soc. 62, 1030-1038.

Lorenzi, M., Bonassi, S., Lorenzi, T., Giovannini, S., Bernabei, R., Onder, G., 2018. A review of telomere length in sarcopenia and frailty. Biogerontology. 19, 209-221.

Martin-Ruiz, C., Jagger, C., Kingston, A., Collerton, J., Catt, M., Davies, K., Dunn, M., Hilkens, C., Keavney, B., Pearce, S.H., 2011. Assessment of a large panel of candidate biomarkers of ageing in the Newcastle 85+ study. Mech Ageing Dev. 132, 496-502.

Martin-Ruiz, C.M., Baird, D., Roger, L., Boukamp, P., Krunic, D., Cawthon, R., Dokter, M.M., van der Harst, P., Bekaert, S., De Meyer, T., 2015. Reproducibility of telomere length assessment: an international collaborative study. Int J Epidemiol. 44, 1673-1683.

Martin-Ruiz, C.M., Gussekloo, J., van Heemst, D., von Zglinicki, T., Westendorp, R.G., 2005. Telomere length in white blood cells is not associated with morbidity or mortality in the oldest old: a population-based study. Aging Cell. 4, 287-290. 
Mather, K.A., Jorm, A.F., Milburn, P.J., Tan, X., Easteal, S., Christensen, H., 2010. No associations between telomere length and age-sensitive indicators of physical function in mid and later life. $\mathrm{J}$ Gerontol A Biol Sci Med Sci. 65, 792-799.

Maynard, S., Keijzers, G., Hansen, Å.M., Osler, M., Molbo, D., Bendix, L., Møller, P., Loft, S., Moreno-Villanueva, M., Bürkle, A., 2015. Associations of subjective vitality with DNA damage, cardiovascular risk factors and physical performance. Acta Physiol (Oxf). 213, 156-170.

Mitnitski, A.B., Graham, J.E., Mogilner, A.J., Rockwood, K., 2002. Frailty, fitness and late-life mortality in relation to chronological and biological age. BMC Geriatr. 2, 1.

Nordfjäll, K., Svenson, U., Norrback, K.-F., Adolfsson, R., Lenner, P., Roos, G., 2009. The individual blood cell telomere attrition rate is telomere length dependent. PLoS Genet. 5, e1000375.

O'Callaghan, N.J., Dhillon, V.S., Thomas, P., Fenech, M., 2008. A quantitative real-time PCR method for absolute telomere length. Biotechniques. 44, 807-809.

Rikli, R.E., Jones, C.J., 1999. Development and validation of a functional fitness test for communityresiding older adults. J Aging Phys Act. 7, 129-161.

Rikli, R.E., Jones, C.J., 2013. Senior fitness test manual. Human Kinetics, Champaign, IL.

Rodier, F., Coppé, J.-P., Patil, C.K., Hoeijmakers, W.A., Muñoz, D.P., Raza, S.R., Freund, A., Campeau, E., Davalos, A.R., Campisi, J., 2009. Persistent DNA damage signalling triggers senescence-associated inflammatory cytokine secretion. Nat Cell Biol. 11, 973-979.

Simm, A., Nass, N., Bartling, B., Hofmann, B., Silber, R.-E., Santos, A.N., 2008. Potential biomarkers of ageing. Biol Chem. 389, 257-265. 
Soares-Miranda, L., Imamura, F., Siscovick, D., Jenny, N.S., Fitzpatrick, A.L., Mozaffarian, D., 2015. Physical Activity, Physical Fitness and Leukocyte Telomere Length: The Cardiovascular Health Study. Med Sci Sports Exerc. 47, 2525-2534.

von Zglinicki, T., Martin-Ruiz, C., 2005. Telomeres as biomarkers for ageing and age-related diseases. Curr Mol Med. 5, 197-203.

Woo, J., Tang, N., Suen, E., Leung, J., Leung, P., 2008. Telomeres and frailty. Mech Ageing Dev. $129,642-648$.

Yu, R., Tang, N., Leung, J., Woo, J., 2015. Telomere length is not associated with frailty in older Chinese elderly: Cross-sectional and longitudinal analysis. Mech Ageing Dev. 152, 74-79.

Zaslavsky, O., Cochrane, B.B., Thompson, H.J., Woods, N.F., Herting, J.R., LaCroix, A., 2013. Frailty: a review of the first decade of research. Biol Res Nurs. 15, 422-432.

Zhou, J., Wang, J., Shen, Y., Yang, Y., Huang, P., Chen, S., Zou, C., Dong, B., 2018. The association between telomere length and frailty: A systematic review and meta-analysis. Exp Gerontol. 106, 1620. 


\section{Figure captions}

Fig. 1. Scatter-plot for univariate associations between baseline telomere length (TL) measured in 2001-2004 and Senior Fitness Test (SFT) results from follow-up in 2011-2013 for women and men.
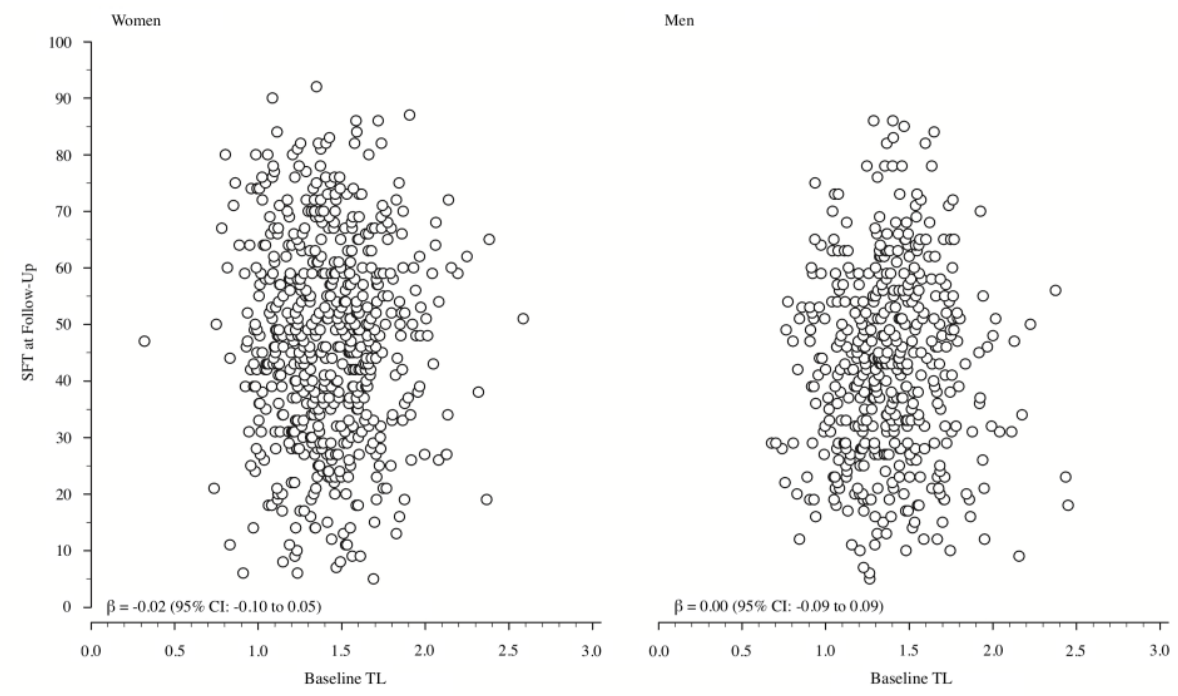

Fig. 2. Scatter-plot for univariate associations between follow-up telomere length (TL) and Senior Fitness Test (SFT) results for women and men.
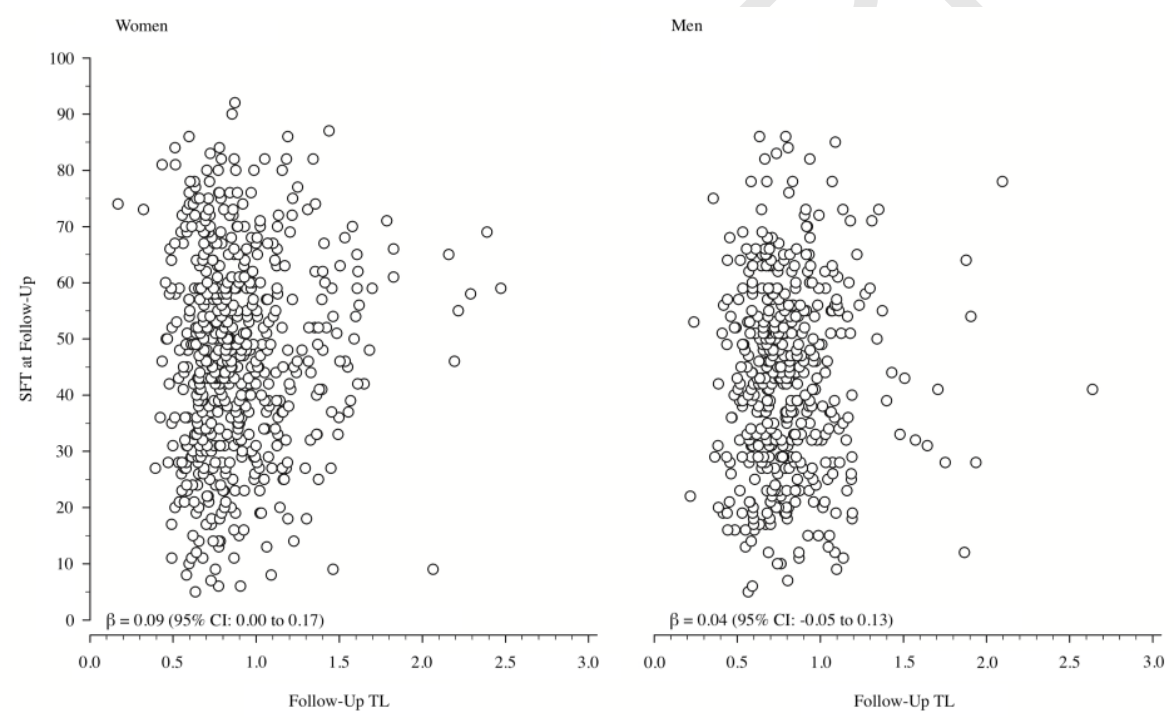

Fig. 3. Relationship between Senior Fitness Test (SFT) results from follow-up in 2011-2013 and relative telomere length at follow-up, measured as follow-up telomere length/baseline telomere length, for women and men. The curves were derived from 3-knot restricted cubic spline regression models. The models were adjusted for age, BMI, education years, smoking and follow-up time. Grey area represents $95 \%$ confidence intervals. 

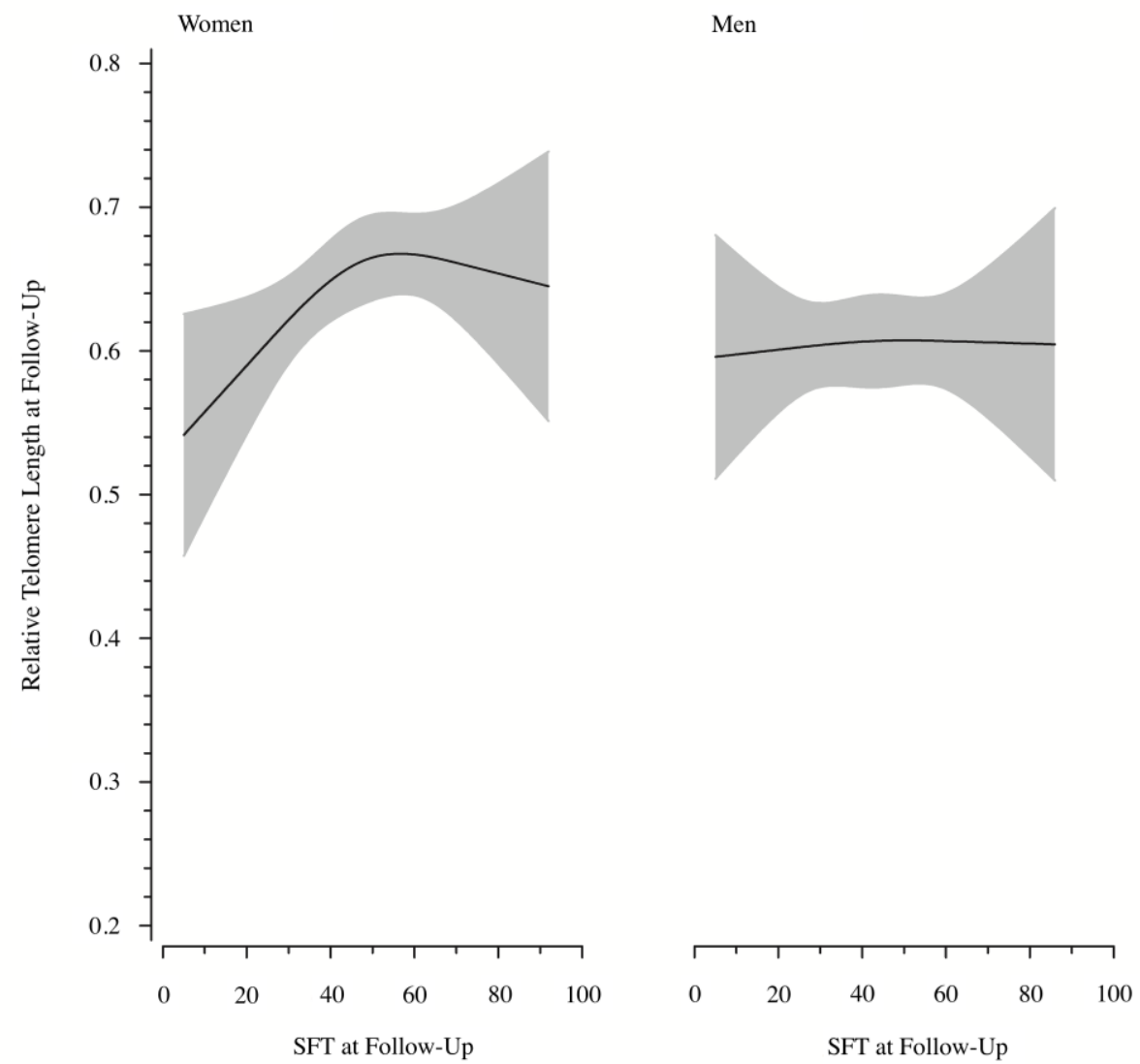
Table 1. Baseline characteristics of cohort members grouped by SFT tertiles at follow-up. Results are presented as means and standard deviations unless otherwise stated

\begin{tabular}{|c|c|c|c|c|c|c|c|c|}
\hline \multirow[b]{2}{*}{ SFT tertile (score range) } & \multicolumn{4}{|c|}{ Women $(n=582)$} & \multicolumn{4}{|c|}{$\operatorname{Men}(n=453)$} \\
\hline & $\mathrm{I}(5-39)$ & II $(40-55)$ & III $(56-100)$ & p-value ${ }^{a}$ & $\mathrm{I}(5-33)$ & II (34-50) & III $(52-100)$ & p-value ${ }^{a}$ \\
\hline $\mathrm{N}$ & 198 & 194 & 190 & $\Delta$ & 149 & 152 & 152 & \\
\hline Age (years) & $61(3)$ & $61(3)$ & $61(3)$ & & $61(3)$ & $61(3)$ & $60(2)$ & \\
\hline Body mass index $\left(\mathrm{kg} / \mathrm{m}^{2}\right)$ & $29.0(5.3)$ & $26.8(4.2)$ & $25.4(3.2)$ & $<0.001$ & $28.4(3.8)$ & $27.2(3.5)$ & $26.1(2.6)$ & $<0.001$ \\
\hline Current smoker or ex-smoker, n (\%) & $95(48)$ & $74(38)$ & $59(31)$ & $<0.001$ & $106(71)$ & $99(65)$ & $106(70)$ & 0.8 \\
\hline \multicolumn{9}{|l|}{ Health status } \\
\hline Cardiovascular disease, $\mathrm{n}(\%)$ & $39(20)$ & $29(15)$ & $15(8)$ & $<0.001$ & $35(23)$ & $30(20)$ & $19(12)$ & 0.01 \\
\hline Diabetes, $\mathrm{n}(\%)$ & $17(9)$ & $4(2)$ & $1(1)$ & $<0.001$ & $22(15)$ & $9(6)$ & $3(2)$ & $<0.001$ \\
\hline Asthma, n (\%) & $14(7)$ & $13(7)$ & $15(8)$ & 0.8 & $14(9)$ & $4(3)$ & $12(8)$ & 0.6 \\
\hline Plasma glucose (mmol/l) & $5.7(1.0)$ & $5.5(1.0)$ & $5.3(0.6)$ & $<0.001$ & $6.4(1.9)$ & $5.9(0.8)$ & $5.7(0.7)$ & $<0.001$ \\
\hline Plasma total cholesterol $(\mathrm{mmol} / \mathrm{l})$ & $6.0(1.1)$ & $5.9(0.9)$ & $6.1(1.0)$ & 0.6 & $5.7(1.1)$ & $5.8(1.0)$ & $5.7(1.0)$ & 0.96 \\
\hline Plasma HDL cholesterol ( $\mathrm{mmol} / \mathrm{l})$ & $1.7(0.4)$ & $1.7(0.4)$ & $1.9(0.5)$ & $<0.001$ & $1.4(0.3)$ & $1.5(0.3)$ & $1.6(0.4)$ & $<0.001$ \\
\hline Plasma triglycerides $(\mathrm{mmol} / \mathrm{l})$ & $1.5(0.7)$ & $1.4(0.6)$ & $1.2(0.6)$ & $<0.001$ & $1.7(0.9)$ & $1.5(0.6)$ & $1.3(0.7)$ & $<0.001$ \\
\hline Education (years) & $11.5(3.4)$ & $12.2(3.4)$ & $12.9(3.4)$ & $<0.001$ & $11.9(3.5)$ & $13.6(3.9)$ & $13.6(3.8)$ & $<0.001$ \\
\hline LTPA (MET-h/year), median (IQR) & $\begin{array}{c}1672(895, \\
2556)\end{array}$ & $\begin{array}{c}1607(966, \\
2561)\end{array}$ & $\begin{array}{c}1983(1218, \\
2853)\end{array}$ & 0.009 & $\begin{array}{c}1506(992, \\
2397)\end{array}$ & $\begin{array}{c}1809(1020, \\
2720)\end{array}$ & $\begin{array}{c}1662(1248, \\
2572)\end{array}$ & 0.1 \\
\hline
\end{tabular}

${ }^{a} \mathrm{p}$-values are calculated for linearity across tertiles

$\mathrm{SFT}=$ Senior Fitness Test; HDL= High-density lipoprotein; LTPA= Leisure-time physical activity; MET= Metabolic equivalent of task; IQR= Interquartile range. 
Table 2. Cross-sectional and longitudinal univariable associations between telomere length and SFT result tertiles.

\begin{tabular}{|c|c|c|c|c|c|c|c|c|}
\hline \multirow[b]{2}{*}{ SFT result (score range) } & \multicolumn{4}{|c|}{ Women $(n=582)$} & \multicolumn{4}{|c|}{$\operatorname{Men}(n=453)$} \\
\hline & $\mathrm{I}(5-39)$ & II $(40-55)$ & III (56-100) & p-value ${ }^{a}$ & $\mathrm{I}(5-33)$ & II $(34-50)$ & III (52-100) & p-value ${ }^{a}$ \\
\hline $\mathrm{N}$ & 198 & 194 & 190 & & 149 & 152 & 152 & \\
\hline \multicolumn{9}{|l|}{ Telomere length (T/S ratio) } \\
\hline Baseline, mean (SD) & $1.42(0.28)$ & $1.41(0.30)$ & $1.42(0.30)$ & 0.87 & $1.37(0.32)$ & $1.38(0.28)$ & $1.39(0.26)$ & 0.45 \\
\hline Follow-up, mean (SD) & $0.85(0.26)$ & $0.91(0.33)$ & $0.92(0.36)$ & 0.021 & $0.80(0.27)$ & $0.80(0.26)$ & $0.82(0.27)$ & 0.56 \\
\hline \multicolumn{9}{|l|}{ Telomere attrition } \\
\hline $\begin{array}{l}\text { Absolute change (T/S ratio), } \\
\text { mean }(95 \% \mathrm{CI})\end{array}$ & $\begin{array}{l}-0.58(-0.62 \text { to }- \\
0.53)\end{array}$ & $\begin{array}{c}-0.50(-0.55 \text { to }- \\
0.44)\end{array}$ & $\begin{array}{c}-0.50(-0.55 \text { to }- \\
0.44)\end{array}$ & 0.039 & $\begin{array}{l}-0.56(-0.62 \text { to }- \\
0.50)\end{array}$ & $\begin{array}{c}-0.58(-0.63 \text { to }- \\
0.52) \\
\end{array}$ & $\begin{array}{c}-0.57(-0.63 \text { to }- \\
0.52) \\
\end{array}$ & 0.86 \\
\hline Relative telomere length ${ }^{b}$ & 0.61 & 0.67 & 0.66 & & 0.60 & 0.60 & 0.61 & \\
\hline
\end{tabular}

${ }^{a} p$-values are calculated for linearity across tertiles

${ }^{\mathrm{b}}$ Calculated as follow-up telomere length/baseline telomere length

$\mathrm{SFT}=$ Senior Fitness Test 
Table 3. Correlation between telomere length and individual SFT components

\begin{tabular}{|l|c|c|}
\hline \multirow{2}{*}{ SFT component } & \multicolumn{2}{|c|}{ Telomere length } \\
\cline { 2 - 3 } & Baseline & Absolute change \\
\hline Women $(\mathrm{n}=582)$ & $\mathrm{r}(95 \% \mathrm{CI})$ & $\mathrm{r}(95 \%$ CI) \\
\hline Arm curl & $0.01(-0.07$ to 0.09$)$ & $0.04(-0.04$ to 0.13$)$ \\
\hline Chair stand & $0.04(-0.04$ to 0.12$)$ & $0.05(-0.03$ to 0.13$)$ \\
\hline Chair sit-and-reach & $-0.01(-0.09$ to 0.08$)$ & $0.04(-0.04$ to 0.12$)$ \\
\hline Back scratch & $-0.05(-0.13$ to 0.04$)$ & $0.06(-0.03$ to 0.14$)$ \\
\hline 6-min walk & $0.01(-0.07$ to 0.09$)$ & $-0.01(-0.09$ to 0.08$)$ \\
\hline Men (n=453) & & $0.00(-0.09$ to 0.09$)$ \\
\hline Arm curl & $0.07(-0.02$ to 0.16$)$ & $-0.06(-0.15$ to 0.03$)$ \\
\hline Chair stand & $0.10(-0.01$ to 0.19$)$ & $0.02(-0.07$ to 0.11$)$ \\
\hline Chair sit-and-reach & $0.02(-0.08$ to 0.10$)$ & $0.02(-0.07$ to 0.11$)$ \\
\hline Back scratch & $-0.02(-0.11$ to 0.07$)$ & $-0.05(-0.14$ to 0.04$)$ \\
\hline 6-min walk & $0.09(-0.01$ to 0.18$)$ & \\
\hline SFT Senior Fitness Test & & \\
\hline
\end{tabular}

SFT= Senior Fitness Test 